\title{
Mães de crianças com cardiopatia congênita: dúvidas e estratégia de intervenção
}

\author{
Mothers of children with congenital heart disease: doubts and intervention strategy \\ Madres de niños con cardiopatía congénita: dudas y estrategia de intervención \\ Maryllia Suellem Almeida Cesario ${ }^{1}$, Alexandre Mansuê Ferreira Carneiro², Maria Fâni Dolabela1*.
}

\begin{abstract}
RESUMO
Objetivo: Descrever os conflitos enfrentados pelas mães de crianças com cardiopatia congênita, bem como apresentar material que pode ser utilizado para o acolhimento destas. Métodos: Trata-se de estudo prospectivo, qualitativo, transversal descritivo, realizado com as mães de recém-nascido cardiopata internado na Unidade de Terapia Intensiva de um Hospital referência no tratamento de crianças com cardiopatia congênita, no período de dezembro de 2018 a julho de 2019. Durante o acolhimento farmacêutico, as mães foram convidadas a participar do trabalho, as que aceitaram foram entrevistadas, em seguida foram retiradas as dúvidas, e sempre que não compreendessem o tratamento medicamentoso, podiam contactar 0 farmacêutico para esclarecer suas dúvidas. Resultados: A maioria das mães possuíam nível de escolaridade médio completo ou incompleto $(64,3 \%)$ residiam no interior do estado $(79,6 \%)$, fizeram pré-natal $(100 \%)$, o diagnóstico foi antes do nascimento (64,2\%) e somente (14,3\%) tiveram o parto prematuro. As mães, em geral, tinham dificuldades em compreender o tratamento, e mostravam-se preocupada quando havia a inclusão de antibióticos ou faltava medicamentos. Visando esclarecer as dúvidas, foi elaborado uma cartilha onde há respostas para as principais questões levantadas pelas mães. Conclusão: Em síntese, é muito importante a preparação das mães de crianças com cardiopatia congênita sobre a doenças, tratamento e dificuldades a serem vencidas.
\end{abstract}

Palavras-chave: Cardiopatia, Mães, Educação em saúde.

\begin{abstract}
Objective: Describe the conflicts faced by mothers of children with congenital heart disease, and present material that can be used to receive them. Methods: This is a prospective, qualitative, descriptive crosssectional study conducted with mothers of newborns with heart disease admitted to Intensive Care Unit at a congenital heart disease reference hospital, from December 2018 to July 2019. During the pharmacy reception, mothers were invited to participate in the study, questions were then answered, and whenever they did not understand about drug treatment, they could contact the pharmacist to clarify their doubts. Results: Most mothers had complete or incomplete high school level (64.3\%) lived in the interior of the state $(79.6 \%)$, all had prenatal care $(100 \%)$, the diagnosis was made before birth $(64,2 \%)$ and only $(14.3 \%)$ had premature birth. Mothers generally had difficulty to understand the treatment and were concerned when antibiotics were included or medications were missing. In order to clarify the doubts, a booklet was prepared where there are answers to the main questions raised by mothers. Conclusion: In summary, it is very important to prepare mothers of children with congenital heart disease about the diseases, treatment and difficulties to be overcome.
\end{abstract}

Keywords: Heart disease, Mothers, Health Education.

\footnotetext{
${ }_{1}^{1}$ Programa de Pós-Graduação em Assistência Farmacêutica, Universidade Federal do Pará (UFPA), Belém-PA.

${ }^{2}$ Centro Universitário do Estado do Pará, Belém, PA. *E-mail: fanidolabela20@gmail.com
} 


\section{RESUMEN}

Objetivo: Describir los conflictos que enfrentan las madres de niños con cardiopatías congénitas, así como presentar material que pueda usarse para recibirlos. Métodos: Este es un estudio transversal, prospectivo, cualitativo y descriptivo, realizado con las madres de recién nacidos cardiopáticos ingresados en la Unidad de Cuidados de un hospital de referencia en el tratamiento de niños con cardiopatía congénita, desde diciembre de 2018 hasta julio de 2019. Durante la recepción de la farmacia, las madres fueron invitados a participar en el trabajo, los que aceptaron fueron entrevistados y respondieron las preguntas y, cuando no entendían el tratamiento farmacológico, podían comunicarse con el farmacéutico para aclarar sus dudas. Resultados: la mayoría de las madres tenían un nivel de escuela secundaria completo o incompleto (64.3\%) vivían en el interior del estado (79.6\%), tenían atención prenatal (100\%), el diagnóstico era antes del nacimiento $(64,2 \%)$ y solo $(14.3 \%)$ tuvieron un parto prematuro. Las madres generalmente tenían dificultades para comprender el tratamiento y les preocupaba la inclusión de antibióticos o la falta de medicamentos. Para aclarar las dudas, se preparó un folleto donde hay respuestas a las principales preguntas planteadas por las madres. Conclusión: En resumen, es muy importante preparar a las madres de niños con cardiopatía congénita sobre las enfermedades, el tratamiento y las dificultades que deben superarse.

Palabras clave: Cardiopatía, Madres, Educación sanitaria.

\section{INTRODUÇÃO}

As malformações congênitas estão entre as principais causas de morte na primeira infância, sendo a cardiopatia congênita (CC) uma das mais frequentes e a de maior morbimortalidade, representando cerca de $40 \%$ das malformações (ROSA RCM, et al., 2013). A incidência de CC varia entre $0,8 \%$ a $1,2 \%$ para cada nascido vivo, nos países desenvolvidos e em desenvolvimento, respectivamente, sendo que no Brasil a taxa é de $1 \%$ (BRASIL, 2017).

Suspeita-se de CC, no período neonatal, quando se observa quatro achados principais: sopro cardíaco, cianose, taquipneia e arritmia cardíaca (AMARAL F, et al., 2002).O diagnóstico precoce, que pode ser realizado ainda intrauterino ou após o nascimento, de preferência nas primeiras 48 horas de vida, contribui para o estabelecimento do tratamento adequado, aumentando a possibilidade de sucesso terapêutico (BRASIL, 2017). Entretanto, na maioria das unidades neonatais, a alta hospitalar ocorre entre 36 e 48 horas após o nascimento, neste período algumas crianças ainda não apresentam sinais clínicos da doença (PAVÃO TCA, et al., 2018)

Esta doença é capaz de comprometer o coração e/ou os grandes vasos sanguíneos levando a danos anatômicos e fisiológicos da criança ainda em desenvolvimento intrauterino. Onde as acianóticas, possuem as comunicações (interatrial, interventricular e persistência do canal arterial - PCA) e as estenoses de valvas (pulmonar e aórtica). Já nas cianóticas, temos a tetralogia de Fallot (TF), a transposição das grandes artérias (TGA), a drenagem anômala de veias pulmonares (DAVP), entre outras. As quais podem ser corrigidas através de procedimento cirúrgico, tendo probabilidade de uma vida normal (FROTA MA, et al., 2014).

Estima-se que 20 a $30 \%$ apresentam defeitos estruturais graves, sendo que 3 a $5 \%$ evoluem a óbito ainda no período neonatal. Alguns casos, devido à falta de diagnóstico precoce, recebem alta hospitalar sem o diagnóstico, e podem evoluir para choque, hipóxia ou óbito precoce, antes de receber tratamento adequado. Este diagnóstico é essencial para a prevenção de agravos e sequelas, melhorando o prognóstico e possibilitando a sobrevida da criança (PAVÃO TCA, et al., 2018).

De forma geral, o recém-nascido (RN) precisa ser hospitalizado em Unidade de Terapia Intensiva Neonatal (UTIN), tendo seu tratamento assistido por uma equipe multiprofissional contando com: médicos, enfermeiros, fisioterapeutas e técnicos de enfermagem, os quais devem estar disponíveis em tempo integral para assistência aos pacientes internados na UTI. Entretanto outros profissionais podem colaborar neste tratamento, sendo assegurados os seguintes serviços à beira leito de assistência: terapia nutricional (enteral e parenteral), fonoaudiológica, psicológica, odontológica, serviço social e farmacêutico (BRASIL, 2010). 
A necessidade de hospitalização do RN, leva seus familiares a terem que conviver em um ambiente desconhecido e diferente ao seu ambiente familiar, com isso precisam se adaptar à nova realidade e buscar formas de enfrentamento, visando diminuir os traumas causados por esta experiência incomum a sua realidade. O familiar ao deparar-se com a situação complexa, precisa compreender o que está acontecendo e deverá lançar mão de suas forças internas/ externas para superar este momento, adaptar-se e prosseguir (BERNARDES AS, 2011). Contudo, além da assistência ao neonato, os profissionais precisam apoiar estes familiares compreendendo suas ansiedades, ter disposição para retirar dúvidas que envolvam aspectos clínicos e o tratamento. Diante disso, o presente estudo descreve os conflitos vividos pelas mães de crianças com CC, bem como apresentar material informativo acerca de CC que pode ser utilizado para o acolhimento destas.

\section{MÉTODOS}

Trata-se de estudo prospectivo, qualitativo, transversal descritivo, realizado com as mães de RN's cardiopatas internados em uma Unidade de Terapia intensiva de um Hospital referência no tratamento de crianças com cardiopatia congênita em Belém do Pará, no período de dezembro de 2018 a julho de 2019.

A UTIN possui apenas 10 leitos e o tempo de internação dos RN's, em geral, excede a 28 dias. O presente estudo foi aprovado no Comitê de Ética em Pesquisa (CAAE; 97704818.8.0000.0018 e parecer: 3.052.776) e autorizada pelo comitê de ética deste Hospital.

Foram incluídas no estudo as mães de RN's com CC internados na Unidade de terapia Intensiva Neonatal (UTIN) do referido hospital, que após o convite e esclarecimentos sobre a pesquisa, concordaram em participar e assinaram o termo de consentimento livre esclarecido (TCLE).

No acolhimento farmacêutico foi realizada uma entrevista estruturada que continha perguntas sobre questões biossociais (idade, escolaridade, local da residência, se fez pré-natal, possui plano de saúde ou se recebia ajuda do governo, se tinha alguma morbidade, se utilizou medicamentos na gestação, se havia histórico de morte súbita na família, e outras informações sobre o diagnóstico da doença no RN). Além disso, as dúvidas sobre o tratamento e doença foram trabalhadas, bem como foi realizada uma escuta qualificada sobre os "sentimentos" das mães em relação a doença e seu tratamento.

Como as questões levantadas pelas mães foram similares, elaborou-se uma cartilha contendo respostas as principais questões apontadas. O objetivo da elaboração desta cartilha foi fornecer a mãe um material com informações qualificadas, com linguagem acessível e que possibilitasse esclarecer as dúvidas de forma rápida.

\section{RESULTADOS}

A amostra foi composta por 14 mães (100\%), que apresentaram o seguinte perfil: a maioria possuía o ensino médio completo ou incompleto $(64,3 \%)$, oriundas do interior do Estado Pará $(78,6 \%)$, sendo que apenas $21,4 \%$ da capital, local onde se localizava o hospital de referência para cardiopatia congênita (CC). e estas mulheres precisaram se deslocar de suas residências para acompanhamento no neonato no período de internação.

Observou-se ainda que uma parte significativa não trabalhava fora do lar (35,7\%), 50\% possuíam diferentes profissões e apenas $14,3 \%$ estudavam. Foram identificadas mulheres com faixa etária entre 17 e 40 anos, ou seja, uma variedade elevada na idade destas mães, mostrando um grau diferenciado de maturidade e conhecimento para aceitação do problema (Tabela 1). 
Tabela 1 - Perfil da mãe incluída no estudo.

\begin{tabular}{ccccc}
\hline Mãe & Idade & Escolaridade & Localidade & Profissão \\
\hline 1 & $17 \mathrm{~A}$ & Médio incompleto & Parauapebas & Do lar \\
2 & $35 \mathrm{~A}$ & Médio completo & Barcarena & Analista de departamento \\
3 & $31 \mathrm{~A}$ & Médio incompleto & Mojú & Do lar \\
4 & $24 \mathrm{~A}$ & Médio completo & Parauapebas & Auxiliar de laboratório \\
5 & $17 \mathrm{~A}$ & Fundamental completo & Tucuruí & Do lar \\
6 & $19 \mathrm{~A}$ & Médio completo & Belém & Do lar \\
7 & $21 \mathrm{~A}$ & Fundamental completo & Bragança & Do lar \\
8 & $29 \mathrm{~A}$ & Médio completo & Macapá & Operador de caixa \\
9 & $29 \mathrm{~A}$ & Superior completo & Capanema & Professora \\
10 & $30 \mathrm{~A}$ & Médio completo & Parauapebas & Atendente \\
11 & $38 \mathrm{~A}$ & Superior completo & Belém & Empresária \\
12 & $27 \mathrm{~A}$ & Médio incompleto & Ananindeua & Estudante \\
13 & $40 \mathrm{~A}$ & Superior Completo & Nova Ipixuna do Pará & Professor \\
14 & $22 \mathrm{~A}$ & Médio completo & Belém & Estudante \\
\hline
\end{tabular}

Legenda: A- anos. Fonte: Cesario MAS, et al, 2019.

Um ponto muito positivo relatado pelas mães, foi $100 \%$ realizaram o pré-natal durante a gravidez, na maioria dos casos foi adotado o parto cesariano e $64,3 \%$ souberam do diagnóstico de C.C antes do nascimento da criança. Dentre estas mulheres com diagnóstico durante a gestação, somente $14,3 \%$ tiveram o parto pré-maturo. Observou-se ainda que, na maioria das gestações o tipo de parto adotado foi o cesariano (85.7\%) e apenas $14,3 \%$ de parto vaginal (Tabela 2).

Tabela 2 - Realização de pré-natal, o tempo de diagnóstico, parto e idade gestacional (IG).

\begin{tabular}{ccccc}
\hline Mãe & Pré-natal & Tipo de parto & IG & Tempo do diagnóstico \\
\hline 1 & Sim & Vaginal & Atermo & Depois \\
2 & Sim & Cesáreo & Termo & Depois \\
3 & Sim & Cesáreo & Termo & Antes \\
4 & Sim & Cesáreo & Pré-termo & Depois \\
5 & Sim & Cesáreo & Termo & Depois \\
6 & Sim & Cesáreo & Termo & Depois \\
7 & Sim & Vaginal & Termo & Antes \\
8 & Sim & Cesáreo & Termo & Antes \\
9 & Sim & Cesáreo & Pré-termo & Antes \\
10 & Sim & Cesáreo & Termo & Antes \\
11 & Sim & Cesáreo & Termo & Antes \\
12 & Sim & Cesáreo & Termo & Antes \\
13 & Sim & Cesáreo & Pré-termo & Antes \\
14 & Sim & Cesáreo & Termo & Antes \\
\hline
\end{tabular}

Fonte: Cesario MAS, et al, 2019

Apenas $14,3 \%$ das mães relataram não ter utilizado nenhum tipo de medicamento durante a gestação e não relataram intercorrência durante a gravidez. Por outro lado, a maioria $(85,7 \%)$ apresentaram diferentes problemas no período gestacional, como por exemplo infecções sexualmente transmissíveis (IST), infecções parasitárias, entre outras. Sendo utilizados medicamentos que não possuem potencial cardiotóxicos e são isentos de prescrição (MIP's), sendo estes prescritos pelo médico. Foi observado que a mãe de número 9 relatou a utilização do ácido retinóico, sendo este um medicamento com potencial cardiogênico (Tabela 3). 
Tabela 3 - Utilização de medicamentos e intercorrências na gravidez relatadas pelas mães

\begin{tabular}{|c|c|c|}
\hline Mãe & Medicamentos & Intercorrências \\
\hline 1 & Creme vaginal, sem descrição do ativo & Leucorréia no $3^{\circ}$ mês \\
\hline 2 & Tioconazol+Tinidazol/ Neutrofer $\AA /$ Regeneses ${ }^{\circledR}{ }^{*}$ & BPA/ sífilis \\
\hline 3 & Ácido fólico & SDI \\
\hline 4 & Sulfato ferroso & Eminência de eclampsia \\
\hline 5 & Sulfato. Ferroso/Ácido fólico & Leucorréia $1^{\circ}$ e $2^{\circ}$ trimestre \\
\hline 6 & Ácido. fólico/ vitaminas/ Dorflex® e Dipirona & IGM +Toxo / IGM + Rubéola** \\
\hline 7 & Sulfato. Ferroso & SDI \\
\hline 8 & Dipirona/Paracetamol/AAS®/ Vitamina /Ômega & Parto cesáreo devido AVP+ CIV \\
\hline 9 & 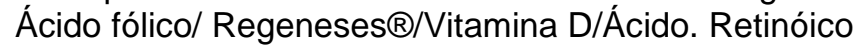 & Pré eclampsia \\
\hline 10 & SDMU & $I G G+T 0 \times 0^{* *}$ \\
\hline 11 & Vitamina C/Regeneses $\AA /$ Sonrisal ${ }^{\circledR}$ & ITU; cesárea devido CC \\
\hline 12 & Materna $\AA^{*} /$ Ácido fólico & ITU tratada \\
\hline 13 & Dramin $\AA /$ Natyben $\AA$ & Gestação anterior- má formação \\
\hline 14 & SDUM & Leucorréia no 3mês \\
\hline
\end{tabular}

Legenda: BPA- bebê em posição anômala; SDI- sem descrição de intercorrência; SDMU- sem descrição de medicamentos utilizados, ITU- infecção do trato urinário; AVP- atresia de valva pulmonar; CIV comunicação interventricular; CC-cardiopatia congênita*polivitamínico; ** através de exames laboratórios foram obtidos resultados positivos para IGM de toxoplasmose, IGM de rubéola ou IGG de toxoplasmose.

Fonte: Cesario MAS, et al, 2019.

Em relação ao acolhimento farmacêutico ao familiar foram apresentadas as atividades que seriam desenvolvidas com a criança durante o período de internação, foi informado que o farmacêutico estaria disponível para esclarecer quaisquer dúvidas dos familiares. Os familiares encontravam-se extremante abatidos com o problema de saúde que acometia o neonato. Um fato interessante foi que, muitas vezes, após o convite para participarem da pesquisa, as mães se recusavam assinar o TCLE, com receio que pudesse ser realizados estudos clínicos de novos medicamentos em seus filhos. Este fato limitou, de forma significativa, o tamanho da amostra.

A religião também foi um fator que interferiu nessa aceitação, em alguns episódios os pais sinalizavam que seu filho(a) não necessitava de outros cuidados, narravam que "Deus sabia o por que seu bebê estaria passando por essa situação". A negativa de mães ao acolhimento do farmacêutico, também provocou interferência na amostra. Elas relataram que não havia necessidade deste serviço, visto que possuía equipe médica na assistência do seu filho. Contudo, elas permitiram o estabelecimento do diálogo e sempre, que possível, foi realizado o esclarecimento das dúvidas e demostrado a importância do farmacêutico neste contexto.

Observou-se ainda que, sempre que ocorria alterações na farmacoterapia por parte da equipe médica, as mães se direcionavam ao farmacêutico solicitando esclarecimentos sobre a mudança, o principal questionamento foi a introdução de antimicrobiano na terapêutica e questionavam: "a bactéria causadora da doença?, É perigosa?; O médico relatou tudo que meu filho tinha?; A medicação é forte?". Outro fator que levou a procura da farmacêutica foi a falta de medicamentos, algumas mães pediram explicações em relação a isto. Além disso, solicitaram esclarecimentos sobre o risco de não utilizar o medicamento que seu filho corria em não utilizar. No caso de falta, muitas se dispuseram em adquirir o medicamento e solicitaram orientações da farmacêutica a este respeito.

Além disso, fizeram outros tipos de questionamentos, como: horário de visita, quem poderia visitar, se poderiam tocar no bebê a qualquer momento, se podiam ou precisavam extrair o leite materno, assim como possuíam dúvidas se poderiam levar itens pessoais de casa para o bebê.

Em síntese, o acolhimento da mãe, permitiu conhecer suas angústias e dúvidas, enquanto, na entrevista pode-se conhecer um pouco sobre sua gestação e utilização de medicamentos neste período. Estas informações contribuíram uma somatória de conhecimento necessário a serem difundidos com essas mães, para isso fez-se necessário a construção e uma cartilha de cuidado farmacêutico para compartilhamento com a equipe multiprofissional e com a família. 
A cartilha tem como objetivo disponibilizar orientações as mães e familiares acerca da CC, a importância dos cuidados recebidos durante a internação na UTIN, assim como os cuidados necessários que devem ser seguidos em ambiente hospitalar durante as visitas. Além disso, informa os horários das refeições, a importância do estabelecimento do diálogo do familiar com a equipe multiprofissional, bem como a necessidade de doação do leite materno e sangue. Outras questões abordadas estão relacionadas com o, sentimento do familiar em relação ao diagnóstico e tratamento da doença. Por fim, a cartilha aborda os cuidados e tratamentos em ambiente domiciliar (Dados Suplementar).

\section{DISCUSSÃO}

Ao analisar os resultados, observa-se que a idade da gestante parece não ter influenciado no nascimento de crianças com CC (Tabela 1). De modo semelhante, outros tem demonstrado que a idade materna não está diretamente relacionada a cardiopatia congênita (FROTA MA, et al., 2014; COSTA DB, et al., 2017). Bem como a idade da mãe parece não ter influenciado no prognóstico do $\mathrm{RN}$, pois os casos fatais estão relacionados a gravidade da má formação e não a idade da mãe (dados não mostrados).

Em relação ao local de domicílio das gestantes, a maioria residia no interior do estado do Pará, todas tiveram acesso ao pré-natal e em $64 \%$ dos casos o diagnóstico ocorreu antes do nascimento da criança (Tabelas 1 e 2). O que não é a mesma realidade de outras mulheres da região Norte, em especial de origem indígena, que têm maior dificuldade em iniciar precocemente o pré-natal (VIELLAS EF, et al., 2014). Historicamente, a mulher tem o papel de provedora de cuidados, no contexto familiar sociocultural e devido a doença terá que abdicar de muitas atividades em prol do RN, gerando vários impactos familiares, em especial quando possui outros filhos considerados incapazes (FROTA MA, et al., 2014).

No presente estudo, em todos os casos a mãe foi o membro familiar mais presente e disponível para o acompanhamento diário dentro do contexto da Unidade de Terapia Intensiva Neonatal (UTIN). Entretanto, outro estudo discute que o cuidado dispensado a criança é atribuído a família a qual ela pertence, no entanto apenas um membro pode assumir o papel de cuidador principal, contribuindo de forma mais direta no cuidado ao neonato e na participação das condições do tratamento. Normalmente essa tarefa é de reponsabilidade da mãe, a qual exerce o papel de realizar o suporte integral e de cuidar nas diversas tarefas e ações durante e também após internação (PAVÃO TL e MONTALVÃO TC, 2016).

Algumas mães, apesar da realização do pré-natal e de exame diagnóstico de Ultrassonografia (US), não houve a detecção da condição clínica do feto (Tabela 2), demonstrando a importância dos cuidados obstétricos que pode contribuir para o diagnóstico precoce e possibilita um melhor planejamento de quando e onde estas crianças devem nascer para receberem uma melhor assistência (MELETI D, et al., 2010).

As mães, em sua maioria, possuíam mais de 9 anos de estudo e apenas 2 possui nível superior. Este pode ser considerado um fato positivo, visto que, quanto maior a escolaridade da mãe maior a possibilidade de compreender a doença e seu tratamento refletindo em um melhor cuidado para a criança (FROTA MA, et al., 2014).

Em relação a grande quantidade de cesarianas detectadas neste estudo, é possível afirmar que os resultados não divergentes de outros autores, pois com aproximadamente $40 \%$ dos partos são cesarianos. $O$ Brasil está entre os países com mais alta taxa deste tipo parto, destacando que o recomendado pela Organização Mundial da Saúde é de apenas 15\% (CAVALCANTE MS, et al., 2012).

Nos países em desenvolvimento a alta taxa de cesariana é um grave e preocupante problema de saúde pública, e demonstra que é muito pequeno o benefício proporcionado ao feto quando se trata de parto cesariano. Esta intervenção pode se vincular a uma maior taxa de óbito materno se comparado ao parto vaginal, variando de quatro a cinco vezes maior, além disso, o parto cesariano encontra-se também associado ao aumento da morbidade e mortalidade perinatal (SOUZA ASR, et al., 2010).

Um dos fatores de risco para as doenças congênitas é o uso de medicamentos, em especial, no início da gestação (BRUM LFS, et al., 2011). No presente estudo, a maioria utilizava antianêmicos, ácido fólico e vitaminas. O resultado do uso de medicamentos no feto pode gerar anomalias no coração, parto prematuro, 
baixo peso ao nascer e atraso no desenvolvimento da criança. Sabe-se também que relativamente poucas drogas causam anomalias congênitas e as estimativas variam quanto à incidência de malformações congênitas que podem ser de até 30\% com varfarina por exemplo e 16\% com valproato de sódio. (MORGAN M, et al., 2011).

Muitos fabricantes de medicamentos a ativos de plantas medicinais desaconselham o uso na gravidez e em lactantes, alegando que não há dados humanos suficientes para demonstrar sua segurança. Evidências para reações adversas transgeracionais a drogas é bastante discutida em relatos de casos. Alguns medicamentos associados a anomalias incluem a varfarina, que pode gerar a síndrome fatal da varfarina, que inclui hipoplasia nasal, defeitos oculares, problemas cardíacos, encurtamento de membros, surdez, escoliose e convulsões. Já a isotretinoína pode gerar defeitos como hidrocefalia, cegueira, problemas faciais, fenda palatina, surdez e defeitos cardíacos. Com o uso de ácido valpróico, pode haver defeitos no tubo neural, defeitos cardíacos, problemas faciais, problemas renais, defeitos nos membros, crânio e músculos. Permanecem desconhecidas as causas da maioria das anomalias congênitas e pode não ser possível obter informações sobre todas as influências supostas (MORGAN M, et al., 2011).

Nos medicamentos atuantes no sistema renina-angiotensina, também há relatos de,um estudo de caso de uma mulher asiática de 30 anos, que esperava seu terceiro filho, sendo os 2 primeiros filhos nasceram saudáveis, ela confirmou que, desenvolveu hipertensão desde a última gravidez e foi tratada pelo clínico geral com antagonista do receptor da angiotensina II (olmesartana). $\mathrm{Na} 20^{\circ}$ semana foi realizado exame de anomalia do bebê e foi detectado uma menor quantidade de líquido amniótico e passado duas semanas, após repetição do exame, foi observada a aparência de nenhum fluido, o exame mostrou também, que os rins estavam normais, mas com a bexiga difícil de delinear, o que sugeria problema com a função renal. Depois de exames adicionais, foi decidido pelos pais a interrupção da gestação devido prognóstico ruim em termos de hipoplasia (MORGAN M, et al., 2011).

O medicamento é contraindicado na gravidez e neste caso seus efeitos podem ter causado os problemas renais do bebê, a recomendação é evitar a prescrição na gravidez devido a efeitos adversos no controle fatal da pressão arterial e na função renal, pois pode causar oligoidrâmnio e possíveis defeitos no crânio. Vários relatos de casos mostraram insuficiência renal em bebês, nos casos em que a mãe utilizou medicamentos deste grupo a por um período maior que 20 semanas de gravidez. A interrupção do uso primeiro trimestre gera um melhor resultado, mas permanece a orientação de evitar a utilização em qualquer momento de gestação (MORGAN M, et al., 2011).

A maioria das mães relataram intercorrências durante a gestação e a mãe número 13 relatou que em gestação anterior houve má formação fetal. Mundialmente, tem sido relatado aumento dos problemas de saúde na gestação, e este fato tem sido relacionado as complexas interações entre fatores demográficos e estilo de vida, além dos avanços da medicina moderna, com novas práticas diagnósticas e terapêuticas. Entre as principais intercorrências clínicas na gravidez, destacam-se: Infecções do Trato Urinário, a Doença Hipertensiva Específica da Gestação e anemia. No Brasil, as principais intercorrências observadas, em gestantes usuários do SUS, foram infecção do trato urinário $(38,2 \%)$, anemia $(26,0 \%)$ e leucorréia $(23,5 \%)$ (VARELA PLR, et al., 2017).

De acordo com Coelho VNPD et al. (2007) o papel do profissional farmacêutico é lutar pelo entendimento da população sobre sua presença e importância nos estabelecimentos, seja qual for seu campo de trabalho e sua especialidade, mostrando-se mais presente e atuante, se tornando um profissional indispensável à saúde pública como um todo (COELHO VNPD, et al., 2007). Neste cenário, a religião das mães pesou negativamente no presente estudo, isto é, mães com maior envolvimento religioso não manifestaram interesse em receber o acolhimento farmacêutico. Este pilar deve ser respeitado por todos os profissionais de saúde, pois a crenças do familiar e paciente são importantes para manter o equilíbrio e a fé. Sendo assim, respeitar as práticas religiosas favorece o equilíbrio emocional, podendo se tornar instrumentos para a recuperação das famílias. Nesse sentido, a religião pode ser uma via de mão dupla, ao mesmo tempo que contribui para o fortalecimento emocional, pode limitar o interesse da busca de informações cientificas (MATIAS LC e RESENDE MC, 2018).

REAS/EJCH | Vol.12(5) | e2337 | DOI: https://doi.org/10.25248/reas.e2337.2020 Página 7 de 9 
Com relação ao tratamento e prognostico dos neonatos com CC acompanhados, foi possível observar que houve abordagens diferenciadas de intervenção, entre estas, introdução do cateterismo, uso de prostaglandina e profilaxia com antibióticos, assim como também houve evolução negativa como introdução de hemodiálise, endocardite bacteriana e sepse.

Ao final observou-se que 4 desses neonatos vieram a óbito, 3 foram transferidos de cuidado pra uti pediátrica e 7 receberam alta hospitalar para acompanhamento ambulatorial. O período de internação foi muito relativo, variou de 7 até 54 dias de internação nesta UTIN, algo bem particular deste cenário, pois o ideal é a permanecia até 28 dias de idade.

\section{CONCLUSÃO}

A existência de apenas 10 leitos para atender este perfil de paciente é um déficit na assistência à saúde, visto que a demanda de outros municípios é evidente e a distância dessas localidades gera uma dificuldade de acompanhamento familiar. Neste contexto é de grande importância a criação de estratégias, como por exemplo o acolhimento farmacêutico que permitiu ouvir essas mães, para poder promover a educação em saúde gerando um melhor entendimento sobre o contexto de saúde do bebê. Para tanto, elaborou-se uma cartilha para amparar as mães neste momento de suas vidas. Por fim esta pesquisa contribui para 0 entendimento e direcionamento de estratégias voltadas para essa população em especial. Contudo, torna-se necessário que haja novas investigações com recorte maior e em outros cenários pelo Brasil.

\section{AGRADECIMENTOS}

A equipe Qualineo e ministério da saúde pela criação e desenvolvimento de um trabalho essencial para melhora da qualidade da assistência ao neonato e sua família. Ao Hospital que permitiu a inclusão do farmacêutico na equipe, ao mestrado profissional em assistência farmacêutica que promoveu 0 desenvolvimento da pesquisa. A toda equipe assistencial que promoveu a inclusão do farmacêutico e troca de saberes que promovem a qualificação do cuidado.

\section{REFERÊNCIAS}

1. AMARAL F, et al. Quando suspeitar de cardiopatia congênita no recém-nascido. Medicina, 2002; 35(2):192--197.

2. BERNARDES, A.S. Cardiopatias congênitas em crianças: aspectos epidemiológicos e manejo da doença. Universidade Castelo Branco atualiza associação cultural enfermagem em cardiologia. Salvador, 2011: 39.

3. BRASIL. Ministério Da Saúde. Síntese de evidências para políticas de saúde: diagnóstico precoce de cardiopatias congênitas. Brasília: Ministério da Saúde; 2017.

4. BRASIL. Ministério da Saúde. Agência Nacional de Vigilância Sanitária - ANVISA. Resolução De Diretoria Colegiada RDC № 7, de 24 de fevereiro de 2010 (Publicada em DOU no 37, de 25 de fevereiro de 2010); 2010.

5. BRUM LFS, et al. Utilização de medicamentos por gestantes usuárias do Sistema Único de Saúde no município de Santa Rosa (RS, Brasil). Ciência \& Saúde Coletiva, 2011; 16(5):2435-2442.

6. CAVALCANTE MS, et al. Complicações maternas associadas à via de parto em gestantes cardiopatas em um hospital terciário de Fortaleza, CE. Revista Brasileira de Ginecologia e Obstetrícia, 2012; 34(3):113-117.

7. COSTA DB, et al. Utilização de medicamentos antes e durante a gestação: prevalência e fatores associados. Caderno de Saúde Pública, 2017; 33 (2): e00126215.

8. COELHO VNPD, et al. O resgate da profissão farmacêutica: Elo entre a saúde e o paciente. Cenarium, 2007: 1-6.

9. FROTA MA, et al. Perfil sociodemográfico familiar e clínico de crianças com cardiopatia congênita atendidas em uma instituição hospitalar. Revista Brasileira de Promoção a Saúde. Fortaleza, 2014; 27(2): 239-246.

10. LIMA GHS, et al. Embriopatia do ácido retinóico: relato de dois casos associados ao uso da isotretinoína. Fisiátrica, $2008 ; 15(1): 59-62$.

11. MATIAS LS, RESENDE MC. Ansiedade e religiosidade de familiares de pacientes internados em unidade de terapia intensiva. Perspectivas em Psicologia, Uberlândia, 2018; 22(1): 38 - 53.

12. MELETI D, et al. A ultrassonografia rotineira em pré-natal de baixo risco colabora com a diminuição das mortalidades maternas e neonatais? Femina, 2010; 38 (8): 435-439. 
13. MORGAN M, et al. Segurança de medicamentos na gravidez - monitorando anomalias congênitas. Journal of Nursing Management, 2011 (19): 305-310.

14. PAVÃO TL, MONTALVÃO TC. Mães Acompanhantes de Crianças Cardiopatas: Repercussões Emocionais Durante a Hospitalização. Revista Psicologia e Saúde, 2016; 8 (2): 67-82.

15. ROSA RCM, et al. Cardiopatias congênitas e malformações extra cardíacas. Revista Paulista de Pediatria, 2013; $31(2): 243-251$.

16. SOUZA ASR, et al. Condições frequentemente associadas com cesariana, sem respaldo científico. Femina, 2010; 38 (10): 505-516.

17. VARELA PLR, et al. Intercorrências na gravidez em puérperas brasileiras atendidas nos sistemas público e privado de saúde. Revista Latino-Americana de Enfermagem, 2017; 25: e2949.

18. VIELLAS EF, et al. Assistência pré-natal no Brasil. Caderno de. Saúde Pública, 2014; 30(1):S1-S15. 